hypothesis of local infectious origin was raised, especially with Proprionibacterium acnes (PA).The possibility of contamination with saprophyte germs coming from the skin during surgery by posterior approach or by epidural infiltration preceding the surgery was also discussed, but not proved.

Objectives: The main objective of this study was to evaluate the prevalence of slow growing bacterias (SGB) in the intervertebral disc (IVD) obtained during a lumbar spine surgery by anterior approach in Modic 1 and 2 changes. A secondary objective was to compare the prevalence of SGB in IVD in lumbar spine surgery obtained by anterior approach to that obtained by posterior approach in herniated disc.

Methods: 45 patients with chronic LBP or sciatica were included in the study, representing 48 IVD. When patients underwent lumbar spine surgery by an anterior approach, 2 samples of the disc were collected for bacteriological analysis: one sample from the anterior part of the disc distant from epidural space and one sample from the posterior part of the disc. 77 discs samples were obtained, 32 discs samples in Modic 1 or 2 changes by anterior approach, 26 discs samples in no Modic IVD by anterior approach, 19 disc samples obtained by posterior approach. The method to collect disc material was strictly aseptic. Samples were analysed by conventional microbial cultures with specialised enrichmen, molecular detection by universal rRNA gene PCR plus sequencing assay. Additionally, all clinical specimens were specifically tested for PA detection using a highly sensitive specific PCR

Results: Regarding bacterial cultures, 12 out 77 disc samples were positive $(16 \%)$, including $10(13 \%)$ for PA. The PA specific PCR was positive for one (1\%) specimen obtained by posterior approach. The 16s RNA detection was positive for 6 specimen (8\%), including one for PA (1\%).Modic 1-2: Cultures were positive in 5 cases $(16 \%)$ with 3 for PA $(10 \%)$. No specific PA PCR was positive. Only one sample was positive for PA in both culture and 16s PCR. Comparison between anterior and posterior approach: Among the PA positive cultures, 5 were identified from anterior specimens $(8.62 \%)$ and 5 from posterior specimens $(26.32 \%)$. Regarding PA cultures, the posterior fragments were more frequently positive than the anterior fragments $(p=0.046)$. The number of epidural infiltrations of the lumbar spine does not seem to influence the bacterial contamination prevalence $p=0.746$. The time between the epidural infiltration of the lumbar spine and the surgery does not seem to influence the bacterial contamination prevalence (more or less 6 month) $p=0.23$.

Conclusions: SGB has been identified in culture in $16 \%$ of the samples obtained in Modic 1 and 2 changes. The prevalence of PA in culture was significantly higher in samples of IVD collected by a posterior approach compared to anterior approach in spine surgery suggesting a contamination. The results of the specific PCR PAs with a single weakly positive sample reinforce the hypothesis of contamination.

Disclosure of Interest: None declared

DOI: 10.1136/annrheumdis-2017-eular.6873

\section{OP0324 MANAGEMENT OF MUSCULOSKELETAL PAIN USING AN ALGORITHM FOR SELECTION OF ANALGESICS}

A. Karateev, A. Tsurgan, N. Gontarenko. V. A. Nasonova Research Institute of Rheumatology, Moscow, Russian Federation

Background: Musculoskeletal pain is the most common manifestation of osteoarthritis $(\mathrm{OA})$ and non-specific back pain (BP). Treatment of pain includes medications with a different mechanism of action such as paracetamol, nonsteroidal anti-inflammatory drugs (NSAIDs), opioid analgesics, muscle relaxants, antidepressants or local glucocorticoid (GC) injections. However, a uniform approach to sequential and complex therapy with these medications is not so far available.

Objectives: The study aims at evaluating the efficacy of combined therapy of musculoskeletal pain in real clinical practice.

Methods: In the open-label study were included 3304 patients (54.3\% women and $45.7 \%$ men with average age of $48.9 \pm 14.6$ years) with acute/subacute pain due to OA or BP. The exclusion criteria were the presence of severe co-morbidities and BP in association with neurological disorders. Treatment was carried out in accordance with the following algorithm: for a moderate/severe pain ( $>4$ scores according to an 11-point numeric rating scale, NRS) use of NSAID (aceclofenac), when NSAIDs are contraindicated - tramadol with/without paracetamol, in case of mild pain - topical NSAID with/without paracetamol, and muscle relaxants as indicated. Control of treatment efficacy was carried out on day 7 (a total of 4 visits). Change of therapy could be done at each visit and include switching to the other NSAID if the prescribed drug proved to be ineffective or intolerant, local glucocorticoid (GC) injection, addition of tramadol with/without paracetamol or administration of antidepressants or anticonvulsants. The results of treatment were assessed based on the dynamics of pain using NRS, a number of patients in whom pain was relieved completely and treatment satisfaction (a 0 to 5 rating scale where 0 is the absence of the effect or pain aggravation and 5 is an excellent effect).

Results: The first prescribed medication in $97.5 \%$ of patients was oral NSAID (aceclofenac $200 \mathrm{mg}$ per day) and in $67.6 \%$ of patients it was aceclofenac in combination with muscle relaxant. By visit 4 , pain decreased from $6.9 \pm 1.5$ to $2.2 \pm 1.3$ points. Pain was completely relieved in $77.0 \%$ of patients. 227 patients $(6.9 \%)$ dropped out of observation, and $16.1 \%$ of patients continued the use of analgesics after four weeks of treatment. The vast majority of patients $(88.4 \%)$ evaluated treatment results as "good" or "excellent". Switching to the other NSAID was required in $8.1 \%$ of patients, local injection of GC in $1.9 \%$, administration of antidepressant or anticonvulsant in $1.5 \%$, and hospitalization in $0.25 \%$ of patients. Adverse reactions (mostly dyspepsia) were noted in $2.2 \%$ of patients.

Conclusions: The use of treatment algorithm based on a complex pathogenetic approach ensures that patients receive an effective and relatively safe pain relief. Oral NSAIDs are the most expedient as first-line treatment in patients with moderate and severe musculoskeletal pain.

Disclosure of Interest: None declared

DOI: 10.1136/annrheumdis-2017-eular.4447

\section{FRIDAY, 16 JUNE 2017}

\section{Regulatory molecules in connective tissue}

\section{OP0325 TGF-BETA-INDUCED ED-A FIBRONECTIN PRODUCTION BY FIBROBLAST-LIKE SYNOVIAL CELLS CONTRIBUTES TO INFLAMMATION IN OSTEOARTHRITIS}

T.W. Kragstrup $^{1,2,3}$, D.H. Sohn ${ }^{1,4}$, C.M. Lepus ${ }^{1}$, K. Onuma ${ }^{1}$, Q. Wang ${ }^{1}$ W.H. Robinson ${ }^{1}$, J. Sokolove ${ }^{1}$. ' ${ }^{1}$ Department of Immunology and Rheumatology, Stanford University, Stanford, United States; ${ }^{2}$ Department of Rheumatology, Aarhus University Hospital; ${ }^{3}$ Department of Biomedicine, Aarhus University, Aarhus C, Denmark; ${ }^{4}$ Department of Microbiology and Immunology, Pusan National University, Pusan, Korea, Republic Of

Background: The pathophysiology of osteoarthritis $(\mathrm{OA})$ involves wear and tear, and a state of low-grade inflammation. Wear and tear leads to tissue degradation followed by tissue repair responses including TGF $\beta$-induced myofibroblast production of extracellular matrix (ECM). Fibronectins are an essential part of the ECM, and injection of fibronectin fragments into rabbit joints is an established animal model of OA. Recently, alternatively spliced fibronectin containing the ED-A domain (ED-A FN) has been shown to activate Toll-like receptor 4

Objectives: In this study, we hypothesize that FN fragments containing the ED-A domain could be one mechanism transducing mechanical events into inflammatory signals in OA.

Methods: Samples of synovial membrane and cartilage were obtained from patients with knee OA undergoing joint replacement surgery. Immunostaining was performed on synovial membranes. Fibroblast-like synovial cells (FLS) isolated by enzymatic digestion of remnant synovial membrane were stimulated with TGF $\beta$, $\mathrm{TNF} \alpha$, lipopolysaccharide, IL-6, OA synovial fluid from two different donors, or chondrocyte lysate, and culture supernatants were analyzed for ED-A FN by immunofluorescence staining. ED-A FN fragments were obtained by plasmin digestion of cellular FN. Synovial cells isolated by enzymatic digestion and human monocyte-derived macrophages (MDM) were incubated with recombinant ED-A FN, plasmin, cellular FN, or cellular FN digested with plasmin; and culture supernatants were analyzed for MCP-1 and TNF $\alpha$.

Results: We hypothesized that ED-A FN is produced by OA FLS in response to products reflecting tear and wear in OA. Indeed, the production of ED-A FN by OA FLS was increased by TGF $\beta$, OA synovial fluid, and lysed chondrocytes in all experiments ( $n=3$, see figure). ED-A FN co-localized with the myofibroblast marker $\alpha$ SMA in both the OA FLS $(n=3)$ and in the OA synovial membranes $(n=8)$. We further hypothesized that ED-A FN expression is associated with inflammation in OA. ED-A FN staining was associated with both number of lining layer cells (rho $=0.85$ and $p=0.011$ ) and infiltrating sublining cells (rho $=0.88$ and $p=0.007$ ) in the OA synovium $(n=8)$, and co-localized with both MCP-1 and TNF $\alpha(n=5)$. Recombinant ED-A FN increased the production of both MCP-1 and TNF $\alpha$ by $\operatorname{MDM}(n=3)$ and OA FLS $(n=3)$. Finally, we demonstrated that the FN fragments containing the ED-A domain generated the same production of both MCP-1 and $\mathrm{TNF} \alpha$ as recombinant ED-A FN.

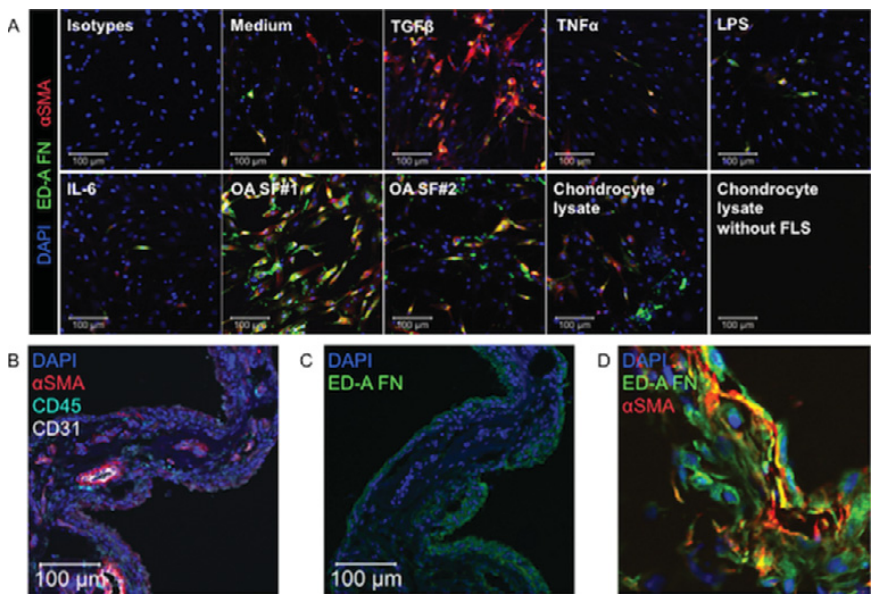

Conclusions: The disease process in $\mathrm{OA}$ shares features with the chronic 
wound healing response including myofibroblast differentiation and that humeral mediators found in the joint can promote myofibroblast production of ED-A FN. We additionally show that recombinant and plasmin-derived ED-A fragments can induce generation of pro-inflammatory mediators from FLS and MDM. This study supports targeting the formation of ED-A FN or the enzymatic fragmentation of FN to reduce pro-inflammatory responses in OA.

Disclosure of Interest: None declared

DOI: 10.1136/annrheumdis-2017-eular.2239

\section{OP0326 EPIGENETICALLY-DRIVEN DISTAL EXPRESSION OF THE LNCRNA HOTTIP SHAPES INFLAMMATORY, ADHESIVE AND PROLIFERATIVE CHARACTERISTICS OF HAND SYNOVIAL FIBROBLASTS IN ARTHRITIS}

M. Frank Bertoncelj ${ }^{1}$, E. Karouzakis ${ }^{1}$, K. Klein ${ }^{1}$, A. Bratus ${ }^{2}$, C. Kolling ${ }^{3}$, O. Distler ${ }^{1}$, A. Filer ${ }^{4}$, R.E. Gay ${ }^{1}$, C.D. Buckley ${ }^{4}$, S. Gay ${ }^{1}$, C. Ospelt ${ }^{1}$.

${ }^{1}$ University Hospital Zurich, Schlieren; ${ }^{2}$ Functional Genomics Center Zurich:

${ }^{3}$ Schulthess Klinik, Zurich, Switzerland; ${ }^{4}$ University of Birmingham, Birmingham, United Kingdom

Background: Rheumatoid arthritis (RA) and other types of inflammatory arthritis show a characteristic pattern of joint involvement. E.g. in RA there is a predilection for the small joints of hands and feet, whereas in spondyloarthropathy, single large joints are characteristically involved. We have recently shown that synovial fibroblasts (SF), which drive joint destruction in RA, display site-specific transcriptomes and functions, shaping unique microenvironments in different joints.

Objectives: To analyze the role of transcripts expressed in SF at specific joint locations in defining location-specific functions of SF, relevant to the pathogenesis of RA.

Methods: SF were isolated from hand, elbow, shoulder, feet, knee and hip joints of RA and OA patients undergoing joint replacement surgery and from knees of nonarthritic subjects with arthralgia. Transcriptomes and epigenomes of SF were determined by RNA-seq (Illumina HiSeq 2000, $n=21$ ), qPCR, ChIPseq (H3K4me3, H3K27me3, H3K27ac, Illumina HiSeq 2500, n=7) and Infinium HumanMethylation450 BeadChip $(n=12)$. Proliferative, adhesive and chemotactic properties of SF were studied by xCELLigence real time cell analysis and leukocyte chemotaxis towards supernatants of SF. The IncRNA HOTTIP was silenced in hand SF using LNA GapmeR oligos, followed by RNA-seq $(n=2)$, pathway enrichment analysis (MetaCore, Thomson Reuters, FDR $<0.05$ ) and gPCR $(n=5)$.

Results: HOTTIP was the most differentially expressed transcript in distal (hand) vs. proximal (shoulder) SF. HOTTIP was specifically transcribed in SF from hand and feet SF, but absent from other joints, inferring distal-specific function to this IncRNA. Hand-specific HOTTIP expression coincided with the enrichment of activating histone marks H3K4me3 and H3K27ac, absence of repressive H3K27me3 and decreased DNA methylation at the HOTTIP promoter in hand SF. In contrast, the HOTTIP promoter displayed abundant DNA methylation and H3K27me3 in knee and shoulder SF. Silencing of HOTTIP led to downregulation of 3275 genes and upregulation of 4326 genes (log ratio $>|0.5|, p<0.01, F D R<0.05$ ). Distal-specific homeobox A13, a known HOTTIP target, was repressed in HOTTIPsilenced SF. Pathway enrichment analysis of genes repressed by HOTTIP silencing showed enrichment for pathways regulating cell adhesion, cell cycle, angiogenesis and inflammation, including NF-kB activation and IL-6 signalling. Meanwhile, upregulated genes were enriched in fewer pathways, e.g. IL17 and Notch signalling. 110 genes that were differentially expressed in hand vs. shoulder SF were also altered by HOTTIP silencing, e.g. TNFRSF1B and MAP3K14. Hand SF showed enhanced proliferative and chemotactic, but decreased adhesive properties compared to shoulder SF.

Conclusions: The IncRNA HOTTIP, which is exclusively expressed in small, distal joints, via epigenetic mechanisms, is a regulator of inflammatory, proliferative and adhesive properties of SF. Such a functional specialization of arthritis relevant pathways in SF might represent an imprinted site-specific "risk" signature in SF, predisposing thereby to location-specific joint pathology, e.g. enhanced severity of hand arthritis in RA.

Disclosure of Interest: M. Frank Bertoncelj Grant/research support from: eurOTEAM, BTCure, IAR, Promedica, Georg und Berta Schwyzer Winiker Grant, E. Karouzakis Grant/research support from: BTCure, GSK, K. Klein Grant/research support from: BTCure, A. Bratus: None declared, C. Kolling: None declared, O. Distler Grant/research support from: Actelion, Bayer, Boehringer Ingelheim, Pfizer, Sanofi, Consultant for: 4 D Science, Actelion, Active Biotec, Bayer, Biogenldec, BMS, Boehringer Ingelheim, ChemomAb, EpiPharm, espeRare foundation, Genentech/Roche, GSK, Inventiva, Lilly, medac, Mepha, Medlmmune, Mitsubishi Tanabe Pharma, Pharmacyclics, Pfizer, Sanofi, Serodapharm, Sinoxa, Speakers bureau: AbbVie, iQone Healthcare, Mepha, A. Filer: None declared, R. Gay Grant/research support from: euroTEAM, BTCure, GSK, IAR, C. Buckley: None declared, S. Gay Grant/research support from: euroTEAM, BTCure, GSK, IAR, C. Ospelt Grant/research support from: euroTEAM, BTCure, CABMM, IAR, Promedica

DOI: 10.1136/annrheumdis-2017-eular.2689
FRIDAY, 16 JUNE 2017

Patient engagement in research: best practices, benefits and challenges

\section{OP0327-PARE YOUR RHEUM - GIVING YOUNG PEOPLE A VOICE IN RHEUMATOLOGY RESEARCH}

K. Cresswell ${ }^{1,2}$, S. Parsons ${ }^{1,2}$, S. Stones ${ }^{3}$, J.E. McDonagh ${ }^{4,5,6}$

W. Thomson $4,5,6$, L. Lunt ${ }^{7} .{ }^{1}$ Public Programmes Team, Central Manchester NHS Foundation Trust, University of Manchester; ${ }^{2}$ Manchester Academic Health Science Centre; ${ }^{3}$ Public Contributor; ${ }^{4}$ Arthritis Research UK Centre for Genetics and Genomics, Centre for Musculoskeletal Research, University of Manchester; ${ }^{5}$ NIHR Manchester Musculoskeletal Biomedical Research Unit, Central Manchester NHS Foundation Trust, University of Manchester; ${ }^{6}$ Barbara Ansell National Network for Adolescent Rheumatology; ${ }^{7}$ Arthritis Research UK Centre for Epidemiology, Centre for Musculoskeletal Research, University of Manchester, Manchester, United Kingdom

Background: Between 2014 and 2016, the Barbara Ansell National Network for Adolescent Rheumatology (BANNAR) commissioned research to explore young people's rheumatology research priorities and beliefs about research involvement. The next phase of this work has been to establish a UK-wide research advisory group, Your Rheum, to involve 11-24 year olds with rheumatic and musculoskeletal diseases (RMDs) more effectively in shaping research.

Objectives: To describe our experiences of developing a UK-wide research advisory group, for young people with RMDs, using both face-to-face meetings and online involvement approaches.

Methods: From September 2016, we recruited young people to the group using several approaches: including the previous research study database, through BANNAR members, through UK charities, such as Arthritis Care and via social media. To tailor options for involvement, young people were recruited to contribute to both face-to-face meetings and via online channels.

Results: Eight young people attended Your Rheum's first meeting in October 2016, where they discussed how they would like the group to work. Thirteen young people have been engaged online via a closed Facebook group, monitored by the Your Rheum facilitator. Key challenges in establishing the group have included developing age-appropriate communication approaches to appeal to the range of ages involved, devising ways of ensuring online members remain engaged with the group, and finding appropriate tasks for the group to be involved with, that are both suitable and aligned with research project timings. This involves working closely with young people, health professionals and researchers.

Conclusions: There is both a need for young people's involvement in research and a desire from young people themselves to do so. Expansion of the online network and involvement activities will allow young people across the UK to have a valuable input into research, regardless of location.

References:

[1] What do young people with rheumatic conditions think about being involved in research? 2017, S.Parsons, W.Thomson, K.Cresswell, B.Starling, J.E.McDonagh (unpublished).

Acknowledgements: This abstract presents independent work funded by Arthritis Research UK BANNAR grant 20164 via the Centre for Adolescent Rheumatology at UCL.

Supported by the National Institute for Health Research Biomedical Research Unit.

We would like to thank all of the young people who took part in the YOURR study and are currently part of Your Rheum, the clinicians, PPI coordinators and other individuals who facilitated their involvement, members of the Barbara Ansell National Network for Adolescent Rheumatology and Arthritis Care.

Disclosure of Interest: None declared

DOI: 10.1136/annrheumdis-2017-eular.5807

\section{FRIDAY, 16 JUNE 2017 \\ Latest advances in the treatment and management of psoriatic arthritis and the latest news on the use of biosimilars in RMDs}

\section{OP0328-PARE PATIENT SAFETY IN RELATION TO BIOSIMILARS - HOW} CAN WE ACT AS A PATIENT ORGANIZATION?

\section{L.M. Thomsen. Danish Rheumatism Association, Gentofte, Denmark}

Background: During the last years two biosimilars has been approved by the national authorities in Denmark, and implemented in the treatment of patients with arthritis. When the first biosimilars were approved in 2015, the hospitals in Denmark decided to shift native patients from the original drug to the new biosimilar. This decision caused considerable insecurity among the patients, who were afraid of biosimilars and their effectiveness and safety profile. Therefore the Danish Rheumatism Association decided to implement an effort to create better patient information and safety for patients, who had to start with a biological drug or shift from one biological drug to another or to a biosimilar. 